\title{
Analysis of potential functional significance of microRNA-3613-3p in human umbilical vein endothelial cells affected by heat stress
}

\author{
JIE LIU ${ }^{1,2}$, XUAN HAN ${ }^{1}$, GUOGUO ZHU ${ }^{1}$, SHIXIN LIU ${ }^{1}$, QIPING LU ${ }^{3}$ and ZHONGZHI TANG ${ }^{1}$ \\ ${ }^{1}$ Department of Emergency, Central Theater General Hospital of People's Liberation Army of China, Wuhan, Hubei 430070; \\ ${ }^{2}$ Department of Emergency, Hefei BOE Hospital Co., Ltd., Anhui, Hefei 230011; ${ }^{3}$ Department of General Surgery, \\ Central Theater General Hospital of People's Liberation Army of China, Wuhan, Hubei 430070, P.R. China
}

Received August 21, 2018; Accepted April 24, 2019

DOI: $10.3892 / \mathrm{mmr} .2019 .10376$

\begin{abstract}
Dysregulation of microRNA-3613-3p (miR-3613-3p) was previously reported in endothelial cells (ECs) during heat stress. The aim of the present study was to investigate the precise role of miR-3613-3p in heat stress. In the present study, potential gene targets of miR-3613-3p in heat-treated ECs were assessed, and the potential effects of miR-3613-3p were determined using Gene Ontology enrichment analysis. Kyoto Encyclopedia of Genes and Genomes pathway analysis was used to identify signaling pathways that may be affected by miR-3613-3p in heat-treated cells. Reverse transcription-quantitative PCR, western blotting and annexin V-FITC/propidium iodide staining were performed to detect miRNA expression, protein expression and apoptosis, respectively. Luciferase gene reporter assay was performed to evaluate the association between miR-3613-3p and mitogen-activated protein kinase kinase kinase 2 (MAP3K2). Bioinformatics analysis revealed 865 potential gene targets for miR-3613-3p and a series of functions and pathways in heat-treated ECs. 'Negative regulation of apoptotic process' was identified as a potential function of miR-3613-3p. In addition, functional analysis confirmed the downregulated expression levels of miR-3613-3p in ECs during heat stress, which was accompanied by an increase in apoptosis; restoration of miR-3613-3p expression inhibited apoptosis. MAP3K2 protein was demonstrated to be upregulated in heat-treated ECs, and overexpression of miR-3613-3p reduced MAP3K2 expression levels. Additionally, MAP3K2
\end{abstract}

Correspondence to: Dr Qiping Lu, Department of General Surgery, Central Theater General Hospital of People's Liberation Army of China, 627 Wuluo Road, Wuhan, Hubei 430070, P.R. China E-mail: jw1293913612@163.com

Dr Zhongzhi Tang, Department of Emergency, Central Theater General Hospital of People's Liberation Army of China, 627 Wuluo Road, Wuhan, Hubei 430070, P.R. China

E-mail: 1293913612@qq.com

Key words: microRNA-3613-3p, heat stress, endothelial cells, bioinformatics, microarray, mitogen-activated protein kinase kinase kinase 2, apoptosis was targeted by miR-3613-3p. These results indicated that miR-3613-3p may have complicated roles in ECs under heat stress. miR-3613-3p may serve an important role in the apoptosis of heat-treated ECs, and this effect may be partly achieved by targeting MAP3K2.

\section{Introduction}

Heat shock (HS) is a life-threatening disorder associated with excessive heat and characterized by a syndrome of multiple organ dysfunction in which central nervous system dysfunction dominates $(1,2)$. The direct cytotoxicity of heat, inflammation and endothelial cell (EC) injury is considered to initiate multiple organ failure (3-5). ECs have a variety of biological functions, including the regulation of coagulation, cell adhesion, nutrient exchange and vascular tone; ECs also serve an important role in the balance of local pro-inflammatory and anti-inflammatory mediators (6-12). Previous studies in cell lines and animal models have shown that ECs are an early target of heat stress injury and are a prominent feature of severe HS $(5,13,14)$. Thus, understanding the changes in ECs in response to heat stress is important for identifying novel therapeutic approaches to HS injury treatment.

MicroRNAs (miRNAs) are small RNAs that regulate gene expression at the post-transcriptional level and modulate biological responses and cell phenotypes through regulation of the expression levels of key proteins in multiple biological pathways (15). Certain miRNAs have been demonstrated to affect the function of ECs. For example, miRNA (miR)-155, miR-320, miR-222, miR-125b, miR-410 and miR-218 have been reported to regulate adherens junction disassembly responses, cell migration and cell morphology, contributing to changes in the permeability and integrity of the vasculature (16-18). As underlying regulatory mechanisms such as miRNA expression in response to HS were unknown, miRNA expression was analyzed in our previous study via miRNA microarrays; it was demonstrated that heat stress altered miRNA expression in HUVECs, with 31 miRNAs differing significantly in expression between HUVECs exposed to severe heat treatment and untreated control cells (20 miRNAs were downregulated and miRNAs were 11 upregulated) (19). Only $1 \%$ of the human miRNA assemblage $(31 / 3,100)$ exhibited differential expression following heat treatment, 
indicating that the regulatory activity of heat stress may be specific toward a subset of miRNAs in HUVECs. Additionally, associations between miRNAs and genes have been previously evaluated on the basis of their differential expression levels, and according to interactions between miRNAs and genes reported in the Sanger miRNA database; these associations were used to build a miRNA-gene network that was employed to evaluate the regulatory effects of miRNAs on genes (19). Using this network model, miR-3613-3p was identified as a key miRNA that may serve a prominent role in the response to heat stress (19).

In the present study, the biological functions and underlying molecular mechanisms of miR-3613-3p in ECs during heat stress were preliminarily investigated. The results may contribute to a better understanding of the mechanisms underlying the relationships between miRNAs and biological responses of ECs and may facilitate new diagnostic and therapeutic strategies for the treatment of heat stress.

\section{Materials and methods}

Bioinformatics analysis. Potential target genes of miR-3613-3p were predicted using three databases: TargetScan 7.2 (http://www.targetscan.org/), miRanda-mirSVR 5.0 (http://www.microrna.org/microrna/home.do) and miRDB 5.0 (http://www.mirdb.org). Functional annotation using Gene Ontology (GO) $(20,21)$ enrichment analysis was performed to determine the main functions of putative target genes of miR-3613-3p. Additionally, Kyoto Encyclopedia of Genes and Genomes (KEGG) (22-24) database analysis was performed to identify the enriched pathways associated with these target genes. Fisher's exact and $\chi^{2}$ tests were applied to assess the significance of GO terms and pathways, and the false discovery rate (FDR) was calculated to correct the P-value. Only GO terms and pathways with an adjusted $\mathrm{P}<0.05$ and an FDR $<0.05$ were selected. Data containing two groups were analyzed using Fisher's exact test, and multiple comparisons between groups were assessed using the $\chi^{2}$ test.

Cell culture and treatment. Human umbilical vein endothelial cells (HUVECs; American Type Culture Collection) were cultured in RPMI 1640 medium (Invitrogen; Thermo Fisher Scientific, Inc.) supplemented with 5\% FBS (Invitrogen; Thermo Fisher Scientific, Inc.), 1\% EC growth supplement (PromoCell GmbH), $100 \mathrm{U} / \mathrm{ml}$ penicillin and $100 \mu \mathrm{g} / \mathrm{ml}$ streptomycin (Invitrogen; Thermo Fisher Scientific, Inc.) in a humidified atmosphere with $5 \% \mathrm{CO}_{2}$ at $37^{\circ} \mathrm{C}$. All experiments were performed using HUVECs at passage 3-6. To induce heat stress, culture dishes containing cells in media were sealed with Parafilm and immersed in a circulating water bath at $43 \pm 0.5^{\circ} \mathrm{C}$ for $1 \mathrm{~h}$; cells in culture dishes placed in a circulating water bath at $37 \pm 0.5^{\circ} \mathrm{C}$ for $1 \mathrm{~h}$ were used as a control. After treatment, the culture medium was replaced with fresh medium, and the cells were incubated at $37^{\circ} \mathrm{C}$ for an additional $24 \mathrm{~h}$.

RNA extraction. Total RNA from HUVECs $\left(\sim 5 \times 10^{6}\right.$ cells) was individually isolated using TRIzol ${ }^{\circledR}$ (Thermo Fisher Scientific, Inc.) and an miRNeasy mini kit (Qiagen, Inc.) according to the manufacturers' protocols. RNA quantity and quality were measured using a NanoDrop ${ }^{\mathrm{TM}}$ spectrophotometer (ND-1000; NanoDrop Technologies; Thermo Fisher Scientific, Inc.) and RNA integrity was detected by gel electrophoresis. In general, RNA concentrations of $8-15 \mathrm{ng} / \mu \mathrm{l}$, and $10 \mathrm{ng} / \mathrm{sample}$ of total RNA were used for subsequent reverse transcription-quantitative PCR (RT-qPCR) analysis.

$R T-q P C R$. The miRNA expression levels were determined using RT-qPCR using primers which were synthesized and purchased from Shanghai GenePharma Co., Ltd. The following primers were used: miR-3613-3p, sense 5'-CGTCCCTTC CCAACCCGAAAAAAA-3', antisense 5'-CGCAGGGTC CGAGGTATTC-3'; and U6, sense 5'-CTCGCTTCGGCAG CACA-3' and antisense 5'-AACGCTTCACGAATTTGCGT-3'. Briefly, sample total RNA (10 ng) was reverse transcribed into cDNA using specific stem-loop primers and a TaqMan ${ }^{\circledR}$ MicroRNA Reverse Transcription kit (Applied Biosystems; Thermo Fisher Scientific, Inc.). Mixtures were incubated for $30 \mathrm{~min}$ at $16^{\circ} \mathrm{C}, 30 \mathrm{~min}$ at $42^{\circ} \mathrm{C}$ and $5 \mathrm{~min}$ at $85^{\circ} \mathrm{C}$, and held at $4^{\circ} \mathrm{C}$ in a $15-\mu 1$ reaction volume. Following the reverse transcription reaction, qPCR was performed using SYBR Green (Invitrogen; Thermo Fisher Scientific, Inc.) according to the manufacturer's protocol and an ABI 7300 Real-Time PCR system (Bio-Rad Laboratories, Inc.). qPCR conditions were $95^{\circ} \mathrm{C}$ for $10 \mathrm{~min}$, followed by 40 cycles of $95^{\circ} \mathrm{C}$ for $15 \mathrm{sec}$ and $60^{\circ} \mathrm{C}$ for $60 \mathrm{sec}$ in a $20 \mu \mathrm{l}$ reaction volume $(25,26)$. Signals were normalized to U6 small nuclear RNA, which was analyzed simultaneously. The relative expression of each miRNA was calculated using the $2^{-\Delta \Delta \mathrm{Cq}}$ method (27). The experiments were performed at least three times.

Transient transfection. The miR-3613-3p mimic (cat. no. miR10017991-1-5) and negative control (miR-NC; cat. no. miR1N0000001-1-5) were purchased from Guangzhou RiboBio Co., Ltd. Cells in the logarithmic growth phase $\left(5 \times 10^{5}\right.$ cells/well) were inoculated into 24 -well plates and cultured to $80 \%$ confluence on the day prior to transfection. Subsequently, miR-3613-3p mimic or miR-NC were transfected into HUVECs at a final concentration of $50 \mathrm{nM}$ using Lipofectamine $^{\circledR} 3000$ reagent (Invitrogen; Thermo Fisher Scientific, Inc.) at room temperature for $24 \mathrm{~h}$ according to the manufacturer's protocol. At $24 \mathrm{~h}$ post-transfection, the transfected cells were harvested for miRNA and protein assays.

Flow cytometric analysis of apoptosis. Apoptosis was analyzed with an Annexin V- FITC Apoptosis kit (Invitrogen; Thermo Fisher Scientific, Inc.) according to the manufacturer's protocol. HUVECs $\left(\sim 1 \times 10^{6}\right.$ cells $)$ were collected, washed with ice-cold PBS and resuspended in binding buffer containing $5 \mu \mathrm{l}$ annexin V-FITC for $10 \mathrm{~min}$ in the dark at room temperature. Subsequently, the cells were pelleted by centrifugation at $\sim 157 \mathrm{x} \mathrm{g}$ and $4^{\circ} \mathrm{C}$ for $10 \mathrm{~min}$, the buffer was removed and the cells were resuspended in reaction buffer containing $10 \mu \mathrm{l}$ propidium iodide (PI). Following incubation in the dark at room temperature for $15 \mathrm{~min}$, the stained cells were analyzed for apoptosis using a FACSCanto ${ }^{\mathrm{TM}}$ II flow cytometer (BD Biosciences). The early apoptotic rates were examined. FlowJo version 7.6.1 software (FlowJo LLC) was used to analyze the data. The experiment was performed at least three times. 


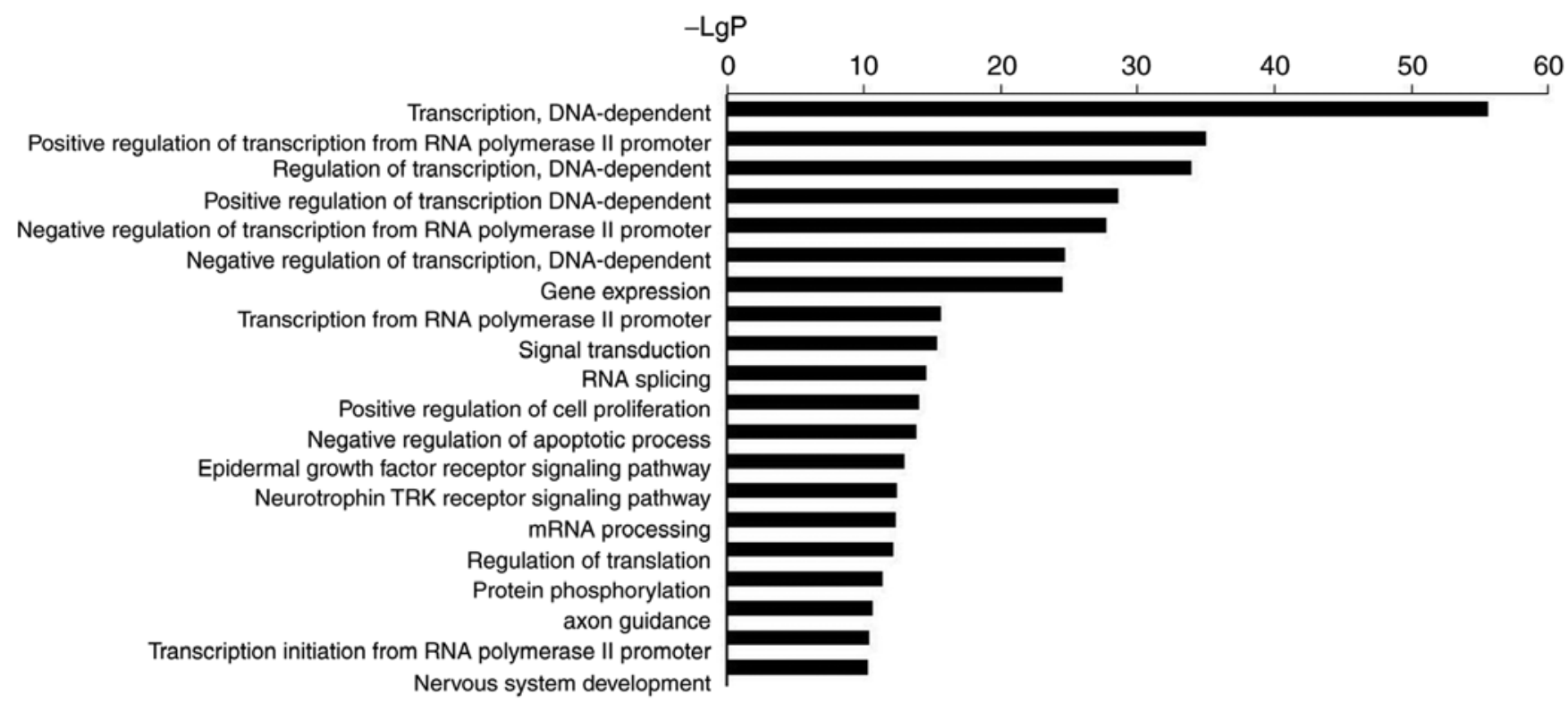

Figure 1. GO term analysis of miR-3613-3p. Top 20 significantly enriched GO biological process functions are presented in descending order. The vertical axis denotes the functional category, and the horizontal axis denotes the degree of enrichment of the function. GO, Gene Ontology; -LgP, negative logarithm of P-value; TRK, tropomyosin-related kinase.

Western blot analysis. HUVECs $\left(5 \times 10^{5}\right.$ cells/well) were seeded in 24-well plates and incubated for $24 \mathrm{~h}$ prior to lysis. Cells were lysed in 1X sample buffer $(62.5 \mathrm{mM}$ Tris, $\mathrm{pH} 6.8 ; 10 \%$ glycerol; 2\% SDS) and homogenized. The protein concentration was measured using a Bicinchoninic Acid Protein assay kit (Thermo Fisher Scientific, Inc.) according to the manufacturer's protocol. Equal amounts of protein $(20 \mu \mathrm{g} / \mathrm{well})$ were separated by $10 \%$ SDS-PAGE and transferred onto polyvinylidene difluoride membranes (EMD Millipore). The membranes were blocked with $5 \%$ non-fat milk powder in PBS $+0.1 \%$ Tween-20 at room temperature for $2 \mathrm{~h}$. The membranes were subsequently incubated overnight at $4^{\circ} \mathrm{C}$ with mouse anti-human mitogen-activated protein kinase kinase kinase 2 (MAP3K2; 1:1,000, cat. no. SAB5300054, Sigma Aldrich; Merck KGaA) or mouse anti-human actin (1:500, cat. no. A4700, Sigma Aldrich; Merck KGaA) primary antibodies. A goat anti-mouse horseradish peroxidase-conjugated immunoglobulin G secondary antibody $(1: 10,000$; cat. no. SAB3701029; Sigma Aldrich; Merck KGaA) was used, with incubation at room temperature for $2 \mathrm{~h}$. Protein bands were visualized with Enhanced Chemiluminescence Western Blot Detection reagent (Thermo Fisher Scientific, Inc.). The membranes were exposed to light-sensitive film and densitometric analysis was conducted using ImageJ v1.50 software (National Institutes of Health). The expression levels of protein were normalized to the actin endogenous control. The western blotting experiments were performed at least three times.

Luciferase gene reporter assay. HUVECs $\left(1 \times 10^{5}\right.$ cells/well) were seeded in 24-well plates and incubated for $24 \mathrm{~h}$ prior to transfection. Cells were co-transfected with $100 \mathrm{nM}$ luciferase vectors (pGL3-MAP3K2 3'UTR wild-type or mutant reporter plasmid; Guangzhou RiboBio Co., Ltd.) and either $50 \mathrm{nM}$ miR-3613-3p mimic or miR-NC using Lipofectamine ${ }^{\circledR} 3000$ at room temperature for $24 \mathrm{~h}$. Firefly and Renilla luciferase activities were quantified using the Dual-Luciferase reporter system (Promega Corporation) according to the manufacturer's protocol. The firefly luciferase activiy was normalized to Renilla luciferase activity. All experiments were performed in triplicate; $n=3-6$ for each experiment.

Statistical analysis. Quantitative variables are presented as the mean \pm standard deviation. Student's t-test was used to calculate statistical significance between two groups. One-way ANOVA was used for multiple group comparisons followed by Tukey post hoc test. Statistical values were calculated using SPSS version 20.0 (IBM Corp.). $\mathrm{P}<0.05$ was considered to indicate a statistically significant difference.

\section{Results}

GO term analysis of miR-3613-3p. GO analysis results were presented in Fig. 1, and Tables I (data repository) and II. According to GO analysis of the 865 genes potentially regulated by miR-3613-3p, the top biological processes associated with the genes regulated by miR-3613-3p were associated with transcription. A number of the genes were also involved in cell proliferation and apoptosis.

KEGG pathway analysis of miR-3613-3p. Pathway analysis by KEGG revealed the signaling pathways affected by miR-3613-3p in heat-treated cells. The most significantly enriched pathways regulated by miR-3613-3p analyzed by KEGG analysis were presented in Fig. 2 and Table III. Enriched KEGG pathway analyses revealed that the top signaling pathways regulated by miR-3613-3p were centralized in cancer-associated terms, which participated in the regulation of apoptosis, proliferation and cell cycle. Additional identified signaling pathways were also associated with transcriptional misregulation in cancer and adherens junctions. 
Table II. Top 20 significant GO biological processes of microRNA-3613-3p target genes.

\begin{tabular}{|c|c|c|c|c|}
\hline GO ID & GO term & Enrichment & P-value & FDR \\
\hline GO:0006351 & Transcription, DNA-dependent & 4.429852641 & $3.20039 \times 10^{-56}$ & $8.225 \times 10^{-53}$ \\
\hline GO:0045944 & $\begin{array}{l}\text { Positive regulation of transcription } \\
\text { from RNA polymerase II promoter }\end{array}$ & 5.752041467 & $1.42286 \times 10^{-35}$ & $1.82837 \times 10^{-32}$ \\
\hline GO:0006355 & $\begin{array}{l}\text { Regulation of transcription, } \\
\text { DNA-dependent }\end{array}$ & 4.150877171 & $1.52829 \times 10^{-34}$ & $1.30924 \times 10^{-31}$ \\
\hline GO:0045893 & $\begin{array}{l}\text { Positive regulation of transcription, } \\
\text { DNA-dependent }\end{array}$ & 6.299381822 & $3.20544 \times 10^{-29}$ & $2.0595 \times 10^{-26}$ \\
\hline GO:0000122 & $\begin{array}{l}\text { Negative regulation of transcription } \\
\text { from RNA polymerase II promoter }\end{array}$ & 6.076613899 & $2.3074 \times 10^{-28}$ & $1.186 \times 10^{-25}$ \\
\hline GO:0045892 & $\begin{array}{l}\text { Negative regulation of transcription, } \\
\text { DNA-dependent }\end{array}$ & 6.125889879 & $2.8019 \times 10^{-25}$ & $1.20015 \times 10^{-22}$ \\
\hline GO:0010467 & Gene expression & 4.938916527 & $4.02629 \times 10^{-25}$ & $1.47822 \times 10^{-22}$ \\
\hline GO:0006366 & $\begin{array}{l}\text { Transcription from RNA polymerase } \\
\text { II promoter }\end{array}$ & 5.640723054 & $3.17439 \times 10^{-16}$ & $1.01977 \times 10^{-13}$ \\
\hline GO:0007165 & Signal transduction & 3.253151632 & $5.30103 \times 10^{-16}$ & $1.51374 \times 10^{-13}$ \\
\hline GO:0008380 & RNA splicing & 6.443742656 & $3.21943 \times 10^{-15}$ & $8.27393 \times 10^{-13}$ \\
\hline GO:0008284 & Positive regulation of cell proliferation & 4.766174616 & $1.15335 \times 10^{-14}$ & $2.69465 \times 10^{-12}$ \\
\hline GO:0043066 & Negative regulation of apoptotic process & 4.366833866 & $1.86122 \times 10^{-14}$ & $3.98612 \times 10^{-12}$ \\
\hline GO:0007173 & $\begin{array}{l}\text { Epidermal growth factor receptor } \\
\text { signaling pathway }\end{array}$ & 6.966208276 & $1.33741 \times 10^{-13}$ & $2.64395 \times 10^{-11}$ \\
\hline GO:0048011 & $\begin{array}{l}\text { Neurotrophin TRK receptor signaling } \\
\text { pathway }\end{array}$ & 5.55742861 & $4.69776 \times 10^{-13}$ & $8.62374 \times 10^{-11}$ \\
\hline GO:0006397 & mRNA processing & 6.873325499 & $5.87456 \times 10^{-13}$ & $1.00651 \times 10^{-10}$ \\
\hline GO:0006417 & Regulation of translation & 12.12939794 & $8.03991 \times 10^{-13}$ & $1.29141 \times 10^{-10}$ \\
\hline GO:0006468 & Protein phosphorylation & 4.727953191 & $5.1723 \times 10^{-12}$ & $7.8193 \times 10^{-10}$ \\
\hline GO:0007411 & Axon guidance & 4.715925224 & $2.91722 \times 10^{-11}$ & $4.16514 \times 10^{-9}$ \\
\hline GO:0006367 & $\begin{array}{l}\text { Transcription initiation from RNA poly } \\
\text { merase II promoter }\end{array}$ & 6.163579932 & $5.51489 \times 10^{-11}$ & $7.45962 \times 10^{-9}$ \\
\hline GO:0007399 & Nervous system development & 4.918192274 & $5.84827 \times 10^{-11}$ & $7.51503 \times 10^{-9}$ \\
\hline
\end{tabular}

FDR, false discovery rate; GO, gene ontology; TRK, tropomyosin-related kinase.

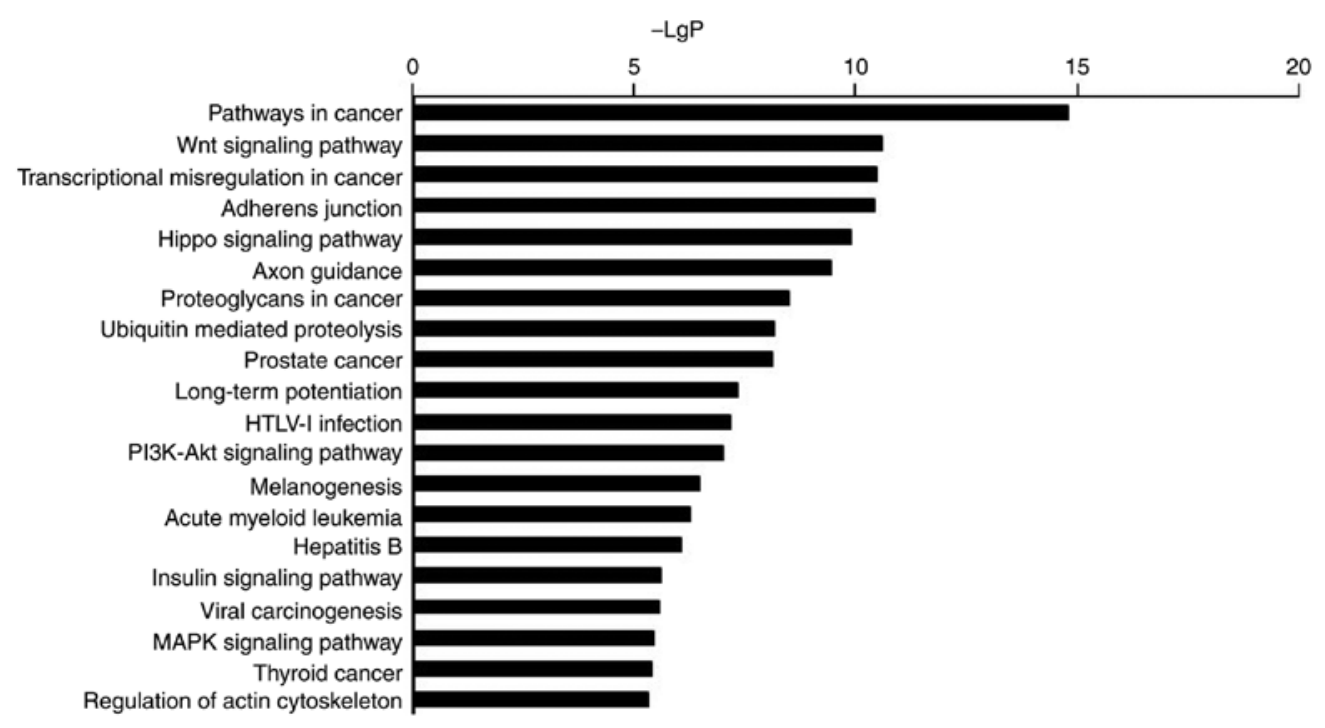

Figure 2. Kyoto Encyclopedia of Genes and Genomes pathway analyses of miR-3613-3p. Top 20 significantly enriched pathways are presented in a descending order. The vertical axis denotes the pathway category, and the horizontal axis denotes the degree of enrichment of the pathway. HTLV, human T-cell leukemia virus; LgP, negative logarithm of P-value; MAPK, mitogen-activated protein kinase. 
Table III. Top 20 significant KEGG pathways of microRNA-3613-3p target genes.

\begin{tabular}{lllll}
\hline KEGG ID & \multicolumn{1}{c}{ Pathway name } & Enrichment & P-value & FDR \\
\hline 05200 & Pathways in cancer & 5.517577809 & $1.69483 \times 10^{-15}$ & $3.25407 \times 10^{-13}$ \\
04310 & Wnt signaling pathway & 7.209781992 & $2.54375 \times 10^{-11}$ & $1.8035 \times 10^{-9}$ \\
05202 & Transcriptional misregulation in cancer & 6.300548374 & $3.54805 \times 10^{-11}$ & $1.8035 \times 10^{-9}$ \\
04520 & Adherens junction & 10.59245368 & $3.75729 \times 10^{-11}$ & $1.8035 \times 10^{-9}$ \\
04390 & Hippo signaling pathway & 6.608966826 & $1.29264 \times 10^{-10}$ & $4.96376 \times 10^{-9}$ \\
04360 & Axon guidance & 7.083198034 & $3.87582 \times 10^{-10}$ & $1.24026 \times 10^{-8}$ \\
05205 & Proteoglycans in cancer & 4.996029548 & $3.30873 \times 10^{-9}$ & $9.07539 \times 10^{-8}$ \\
04120 & Ubiquitin mediated proteolysis & 6.350355081 & $7.1811 \times 10^{-9}$ & $1.63216 \times 10^{-7}$ \\
05215 & Prostate cancer & 8.108979522 & $7.65076 \times 10^{-9}$ & $1.63216 \times 10^{-7}$ \\
04720 & Long-term potentiation & 8.712666126 & $4.7221 \times 10^{-8}$ & $9.06642 \times 10^{-7}$ \\
05166 & HTLV-I infection & 4.231711595 & $7.15157 \times 10^{-8}$ & $1.24827 \times 10^{-6}$ \\
04151 & PI3K-Akt signaling pathway & 3.713972712 & $1.06017 \times 10^{-7}$ & $1.69628 \times 10^{-6}$ \\
04916 & Melanogenesis & 6.635140952 & $3.41775 \times 10^{-7}$ & $5.04776 \times 10^{-6}$ \\
05221 & Acute myeloid leukemia & 9.043849341 & $5.42797 \times 10^{-7}$ & $7.44407 \times 10^{-6}$ \\
05161 & Hepatitis B & 5.224656207 & $8.84732 \times 10^{-7}$ & $1.13246 \times 10^{-5}$ \\
04910 & Insulin signaling pathway & 5.154994125 & $2.62577 \times 10^{-6}$ & $3.15092 \times 10^{-5}$ \\
05203 & Viral carcinogenesis & 4.233570054 & $2.90592 \times 10^{-6}$ & $3.28198 \times 10^{-5}$ \\
04010 & MAPK signaling pathway & 3.767111091 & $3.92363 \times 10^{-6}$ & $4.1852 \times 10^{-5}$ \\
05216 & Thyroid cancer & 12.44308927 & $4.33983 \times 10^{-6}$ & $4.38551 \times 10^{-5}$ \\
04810 & Regulation of actin cytoskeleton & 4.076041866 & $4.92093 \times 10^{-6}$ & $4.7241 \times 10^{-5}$ \\
\hline
\end{tabular}

FDR, false discovery rate; HTLV, human T-cell leukemia virus; KEGG, Kyoto Encyclopedia of Genes and Genomes; MAPK, mitogen-activated protein kinase.

miRNA expression levels are dysregulated in HUVECs during heat stress. Based on previous miRNA microarray analysis, miR-3613-3p expression levels were decreased in HUVECs under heat stress (19). To confirm the downregulation of miR-3613-3p in heat-treated cells, miR-3613-3p expression was analyzed by RT-qPCR, and the results verified that miR-3613-3p expression was reduced in HS-treated HUVECs compared with control cells $(\mathrm{P}<0.05$; Fig. 3 ).

miR-3613-3p affects apoptosis in HUVECs during heat stress. Previous bioinformatics analysis demonstrated that miR-3613-3p negatively regulates apoptosis (19). To confirm this, miR-3613-3p mimic was transfected into HUVECs, which increased the expression levels of microRNAs-3613-3p (Fig. 4A). Annexin V-FITC/PI flow cytometry was then used to evaluate the effects of different expression levels of miR-3613-3p on the apoptotic rates of HUVECs. The results revealed that miR-3613-3p expression decreased in HUVECs following heat stress and that the miR-3613-3p mimic increased the level of miR-3613-3p in heat-treated and unheated cells ( $\mathrm{P}<0.05$; Fig. 4A). In addition, heat stress induced apoptosis in HUVECs, whereas the miR-3613-3p mimic transfection significantly decreased the apoptotic rate under heat stress $(\mathrm{P}<0.05$; Fig. 4B). These results suggested that the level of miR-3613-3p may affect HUVEC apoptosis during heat stress.

miR-3613-3p affects the expression of MAP3K2 in HUVECs during heat stress. Previous bioinformatics analysis indicated

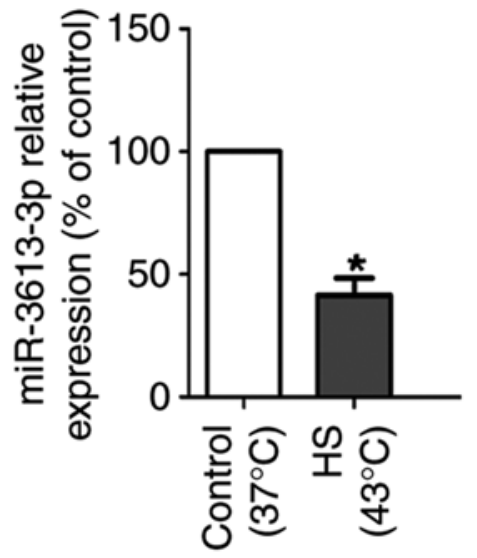

Figure 3. Expression of miR-3613-3p is dysregulated in HUVECs during heat stress. Reverse transcription-quantitative PCR analysis was performed to analyze miRNA expression levels. miR-3613-3p was significantly downregulated following heat shock compared with the control group. ${ }^{*} \mathrm{P}<0.05$ vs. control $\left(37^{\circ} \mathrm{C}\right)$. HS, heat shock; HUVECs, human umbilical vein endothelial cells; miR, microRNA; miRNA, microRNA.

that MAP3K2 may be a target gene of miR-3613-3p (19). To investigate the association between miR-3613-3p and MAP3K2, HUVECs were transfected with miR-3613-3p mimic for $24 \mathrm{~h}$, and western blotting was performed. As presented in Fig. 5, the protein level of MAP3K2 was significantly reduced in the miR-3613-3p mimic-transfected cells under heat treatment, compared with cells only treated 

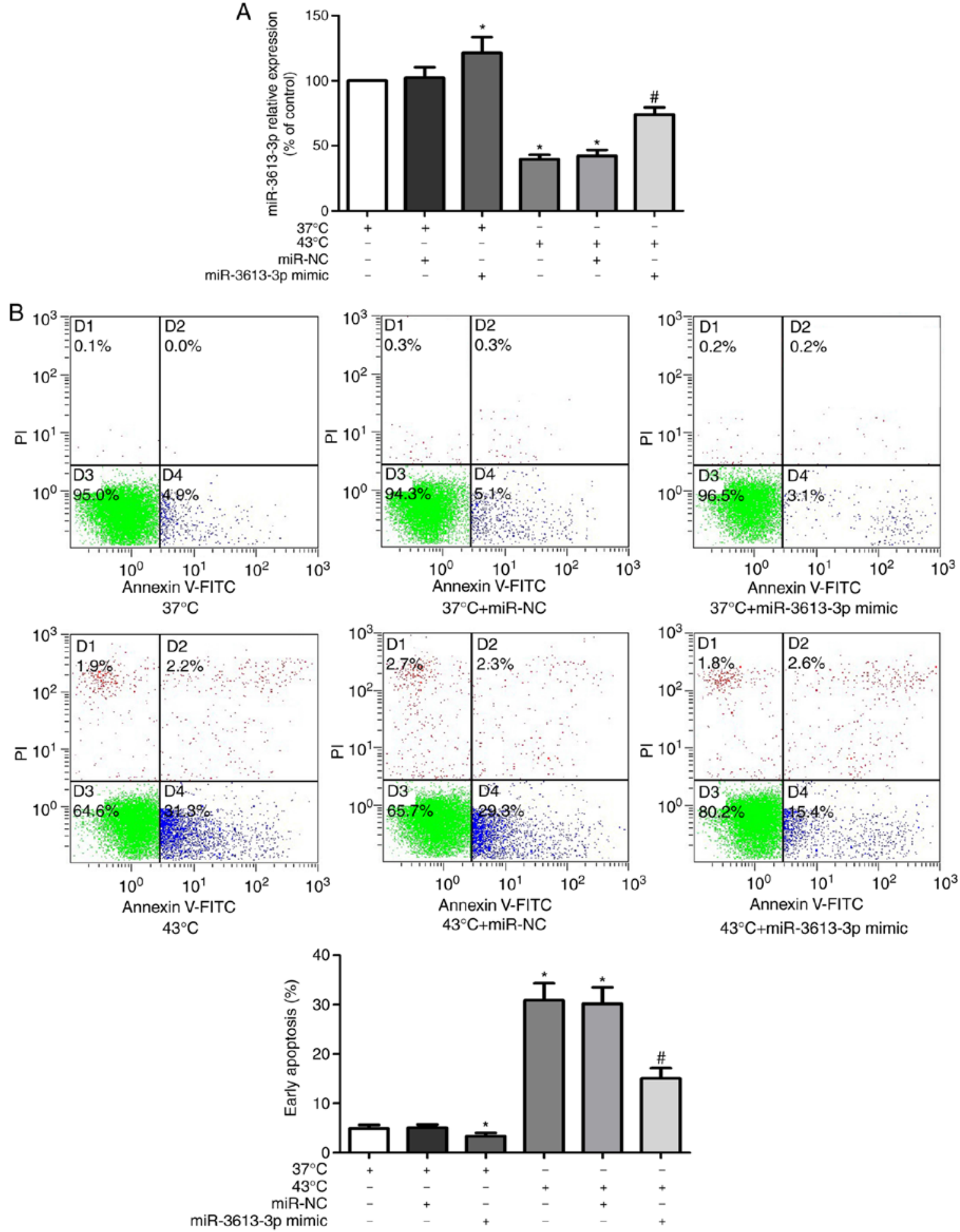

Figure 4. miR-3613-3p affects apoptosis in HUVECs during heat stress. (A and B) HUVECs were transfected with a miR-3613-3p mimic or miR-NC. (A) Expression of miR-3613-3p was decreased in HUVECs under heat stress, and the miR-3613-3p mimic partly reversed the heat stress-induced decline in miR-3613-3p. (B) Annexin V-FITC/PI flow cytometry was used to detect the effect of miR-3613-3p on the apoptotic rate of HUVECs. Heat stress induced apoptosis in HUVECs, and the miR-3613-3p mimic partially reversed the effects of heat stress. ${ }^{*} \mathrm{P}<0.05$ vs. $37^{\circ} \mathrm{C} ;{ }^{*} \mathrm{P}<0.05$ vs. $43^{\circ} \mathrm{C}$. HUVECs, human umbilical vein endothelial cells; miR, microRNA; NC, negative control; PI, propidium iodide.

with heat stress $(\mathrm{P}<0.05)$. These results indicated that miR-3613-3p overexpression may downregulate MAP3K2 expression.

MAP3K2 is a direct target of miR-3613-3p in HUVECs. To further demonstrate whether MAP3K2 was a direct target of miR-3613-3p, MAP3K2 wild-type and mutant 3'UTR were cloned into a luciferase reporter vector and transfected into HUVECs (Fig. 6A). The effects of miR-3613-3p were determined using the luciferase reporter assay. The results demonstrated that upregulation of miR-3613-3p significantly reduced the luciferase activity of pGL3-MAP3K2 3'UTR WT 


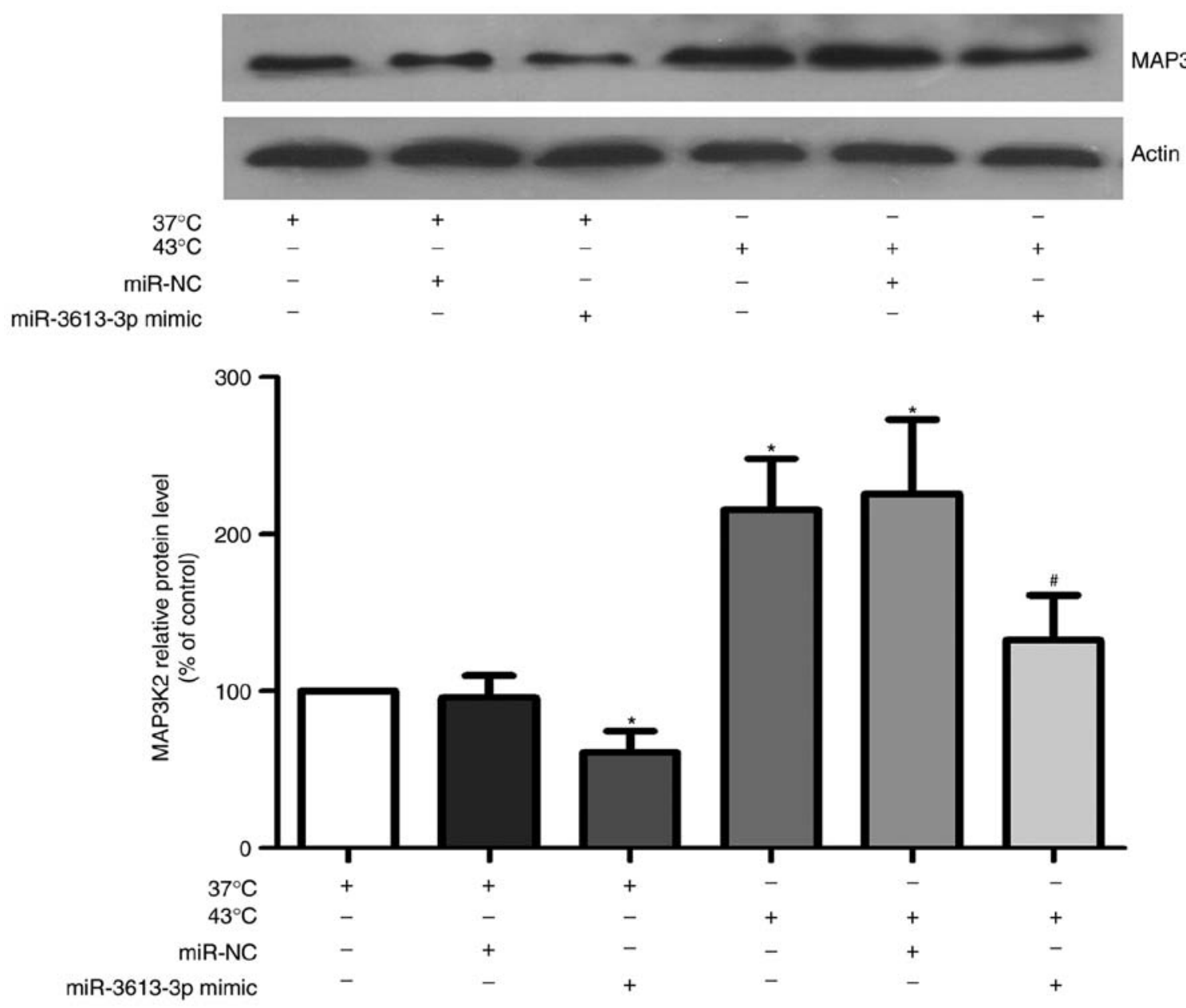

Figure 5. miR-3613-3p affects the expression of MAP3K2 in HUVECs during heat stress. HUVECs were transfected with a miR-3613-3p mimic or miR-NC, and expression of MAP3K2 was analyzed by western blotting using actin as an internal control. MAP3K2 protein expression was increased in heat-treated HUVECs, whereas upregulation of miR-3613-3p partly reversed the increase in MAP3K2 induced by heat stress. ${ }^{*} \mathrm{P}<0.05$ vs. $37^{\circ} \mathrm{C} ;{ }^{\prime \prime} \mathrm{P}<0.05$ vs. $43^{\circ} \mathrm{C}$. HUVECs, human umbilical vein endothelial cells; MAP3K2, mitogen-activated protein kinase kinase kinase 2; miR, microRNA; NC, negative control.

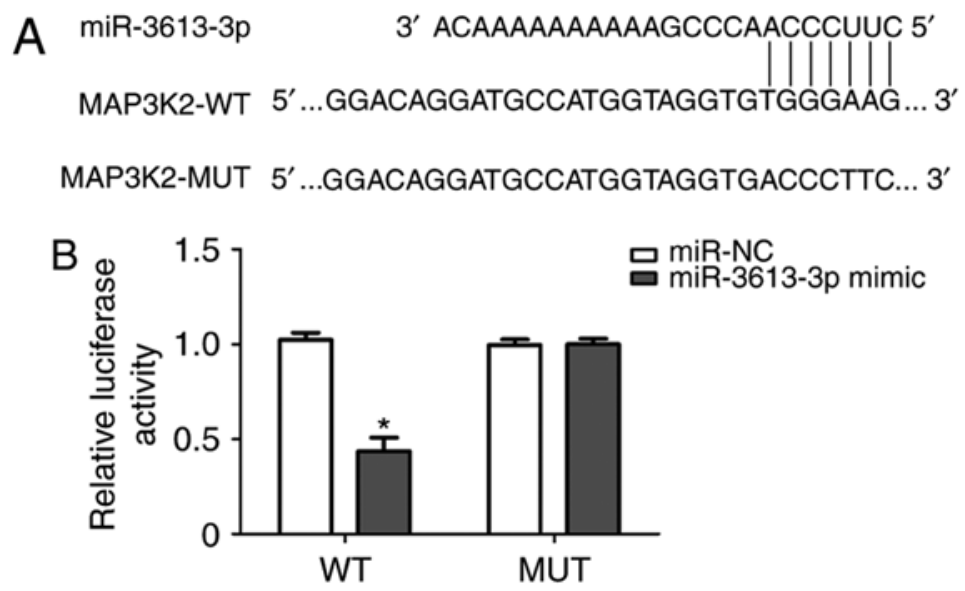

Figure 6. MAP3K2 is a target gene for miR-3613-3p. (A) Schematic representation of the putative miR-3613-3p target site in the MAP3K2 3'untranslated region. (B) Analysis of relative luciferase activity of MAP3K2-WT, MAP3K2-MUT in HUVECs. "P<0.05 vs. miR-NC + WT. HUVECs, human umbilical vein endothelial cells; MAP3K2, mitogen-activated protein kinase kinase kinase 2; miR, microRNA; MUT, mutant; WT, wild type.

$(\mathrm{P}<0.05)$, but the effects of miR-3613-3p was not observed with the mutation of the miR-3613-3p target site in the MAP3K2 3'UTR (Fig. 6B). These results suggested that MAP3K2 may be directly and negatively regulated by miR-3613-3p.

\section{Discussion}

Among the miRNAs identified and target genes predicted in our previous study, miR-3613-3p was identified as an miRNA 
downregulated in heat-treated cells compared with non-treated control (19); it was also demonstrated to potentially regulate 865 genes. Similar results were observed in a previous bioinformatics prediction, which suggested that miRNAs regulate $>30 \%$ of protein-coding genes and that one miRNA may have hundreds or even thousands of potential gene targets (15). Indeed, the wide target range of miRNAs appears to be an important biological mechanism that enables them to mediate different cellular responses to environmental changes and is likely to significantly advance our knowledge of miRNA biological functions. For example, several studies have demonstrated that miR-3613-3p is upregulated in lung adenocarcinoma (28) and in gastric (29), colon (30), prostate (31) and thyroid papillary (32) cancers. In addition, the high level of miR-3613-3p expression in gastric cancer cells was reduced by cinobufagin (33), which is used clinically to treat patients with solid malignant tumors. Thus, miR-3613-3p might serve an important role in cancer by acting as an oncogene. In addition, by targeting pain-associated genes, including $\gamma$-aminobutyric acid receptor type A subunit $\beta 3, \mathrm{~N}$-methyl-D-aspartate $3 \mathrm{~A}$, transient receptor potential vanilloid-1, neuropeptide $Y$ receptor $\mathrm{Y} 1$, and sodium channel protein type 9 subunit $\alpha$, miR-3613-3p may be involved in severe axial pain after motor vehicle collision in African Americans (34). Upregulation of miR-3613-3p was observed in the left atrial appendage in rheumatic mitral valve disease with atrial fibrillation (35). Another report indicated that decreased expression of miR-3613-3p may regulate $B$ cell activation through the Wnt pathway and participate in the pathogenesis of immunoglobulin A nephropathy (36). Therefore, it was speculated that miR-3613-3p may have different functions and regulate different target genes in various tissues under different conditions. This may also explain the large number of target genes of miR-3613-3p. Overall, the effects of miR-3613-3p in ECs under heat stress need to be clarified.

According to GO analysis, there was potential significant enrichment of miR-3613-3p in transcription-related functions (8 of 10 terms, $80 \%$ ), and RNA polymerase II may be a key regulatory point. This finding was expected as the transcriptional status of cells changes markedly in response to heat stress. Transcriptional activity is commonly considered to decrease globally with transcriptional activation of certain genes that are necessary for heat stress-related cellular responses (37). However, there is no clear understanding of the exact mechanism of transcriptional repression. RNA polymerase II activity reduction is mediated by Alu RNAs, which are non-coding RNAs representing transcripts of short interspersed elements (SINE) in humans (38). Under heat stress, Alu RNAs are rapidly upregulated $(39,40)$ and bound directly to RNA polymerase II to form kinetically stable complexes, thereby interfering with the binding of RNA polymerase to DNA and subsequent formation of the closed complex (41). To mediate repression, small repressing RNAs bind to RNA polymerase prior to formation of the closed complex (42), which prevents the mechanism from interfering with the transcription of heat shock protein (HSP) genes that are associated with RNA polymerase II molecules under non-heat shock conditions $(43,44)$; this results in HS-induced increase in HSP expression despite a global decline in cell transcription. However, it is unclear whether SINE-dependent transcriptional repression is universal in cells under heat stress. The data from the present study suggested that miR-3613-3p may perform a complex regulatory function in transcription during heat stress. miR-3613-3p may be involved in regulating a variety of transcription-related processes and appears to simultaneously regulate opposing functions. For example, miR-3613-3p was found to be involved not only in 'positive regulation of transcription' but also in 'negative regulation of transcription'. It is not clear how miR-3613-3p participates in the regulation of transcription and whether it depresses transcription via SINE-dependent mechanisms. Thus, miR-3613-3p may serve a complex role in the regulation of transcription through unknown and complicated mechanisms.

KEGG pathway analysis provided a more detailed view of the integrated relationship between the regulation of miR-3613-3p and target genes. The pathways identified by KEGG analysis serve crucial roles in regulating diverse processes of ECs, including proliferation, apoptosis, survival, migration and polarity, tight junction integrity and monocyte-EC adhesion enhancement, mostly through a series of downstream transcription factors (45-53). These data suggested that miR-3613-3p may facilitate individual biological reactions not by targeting an isolated pathway, but rather by targeting a complete network.

Through the bioinformatics analysis in the present study, 'negative regulation of apoptotic process' was identified as a potential function of miR-3613-3p. Apoptosis is implicated in the physiological and pathological processes of ECs under heat stress (54-56). In vitro experiments on the inhibitory effect of miR-3613-3p on apoptosis indicated that heat stress induced miR-3613-3p repression and promoted apoptosis. In addition, reintroduction of $\mathrm{miR}-3613-3 \mathrm{p}$ partly reversed the promoting effect of heat stress on apoptosis, which suggested that miR-3613-3p may be involved in regulating the apoptotic process in ECs during heat stress; these results may provide new insight into the treatment of HS.

Our previous miRNA-gene network analysis revealed a series of hub target genes, and MAP3K2 appeared in the central position of the network regulated by many miRNAs (19). MAP3K2 is a component of the mitogen-activated protein kinase (MAPK) signaling pathway, which preferentially regulates JNK and ERK5 pathways by phosphorylating and activating MAP2K5 and MAP2K7 (57,58). In addition, MAP3K2 participates in the regulation of apoptosis through a number of downstream targets $(59,60)$. In the present study, bioinformatics analysis indicated that MAP3K2 was a target gene of miR-3613-3p. Therefore, miR-3613-3p may be involved in EC apoptosis by targeting the MAP3K2 gene during heat stress. In the current study, increased expression of MAP3K2 was observed in ECs under heat stress accompanied by reduced expression of miR-3613-3p compared with non-treated cells, whereas overexpression of miR-3613-3p partly reversed MAP3K2 augmentation, which suggested that MAP3K2 may be partially regulated by miR-3613-3p. Additionally, miR-3613-3p directly inhibited the expression of MAP3K2 by binding to its 3'UTR. It is noteworthy that MAK3P2 may induce distinct effects on apoptosis under varying external conditions (61-63), which may be due to distinct downstream molecules. The MAP2K5/ERK5 pathway is required for normal cardiovascular development and 
vascular integrity, and it improves EC viability and reduces apoptosis $(64,65)$; conversely, JNK MAPKs promote apoptosis in ECs under most conditions (66). MAK3P2 coordinately activates signaling through MEK5/ERK5 and MEK7/JNK protein kinases to respond to different stimuli. Therefore, the effects of MAP3K2 on EC apoptosis during heat stress may be induced by JNK MAPKs. The present study demonstrated that heat stress may decrease the activation of miR-3613-3p and promote the apoptotic effect on HUVECs by MAP3K2 through targeted binding to 3'-UTR, which leads to the inhibition of MAP3K2 expression.

There were certain limitations to the present study. First, HUVECs cannot completely reflect the biological characteristics of vascular ECs as venous endothelial function may differ substantially from arterial or capillary endothelial function. It remains to be determined whether similar regulatory mechanisms are involved in vivo and in other cellular systems. Second, the heat stress cell model used in the present study did not ideally simulate the clinical course of HS, and interactions between ECs and other cells were ignored. Third, some of the bioinformatics results may have been affected by technical deficiencies, such as technical artifacts introduced by the smaller scale of experimentally validated data and other associated biological data, deficiencies in effective integration of diverse datasets or the different computational models $(67,68)$.

In conclusion, bioinformatics analysis revealed a range of target genes of miR-3613-3p, and a number of functions and pathways involved. Additionally, "negative regulation of apoptotic process' was predicted to be a potential function of miR-3613-3; in vitro analysis revealed that miR-3613-3p may suppress apoptosis in ECs under heat stress, potentially by directly targeting MAP3K2. These results shed new light on the underlying mechanisms of heat stress in ECs, although further studies are needed to explore the roles of miR-3613-3p in the pathogenesis of heat stress in ECs.

\section{Acknowledgements}

The authors would like to thank Dr Cuini Wang (Department of Immunology, Shanghai Medical College, Institute for Immunobiology of Fudan University) for her advice and our medical writer for their help in preparing the manuscript.

\section{Funding}

No funding was received.

\section{Availability of data and material}

The datasets used and/or analyzed during the current study are available from the corresponding author on reasonable request. Table I is published in the following repository: https://figshare.com/s/81268025c7dac8b67bd2 (DOI:10.6084/m9.figshare.7435958).

\section{Authors' contributions}

QL and ZT conceived and designed all the experiments, performed data analysis for the study, and contributed towards overall supervision of the project, and the drafting and editing the manuscript. JL was involved in the design of the study, performed experiments and data analysis, and was a major contributor to the writing of the manuscript. SL and GZ analyzed and interpreted the data regarding miR-3613-3p. $\mathrm{XH}$ performed reverse transcription-quantitative PCR for miR-3613-3p. All authors read and approved the final manuscript.

\section{Ethics approval and consent to participate}

Not applicable.

\section{Patient consent for publication}

Not applicable.

\section{Competing interests}

The authors declare that they have no competing interests.

\section{References}

1. Robine JM, Cheung SL, Le Roy S, Van Oyen H, Griffiths C, Michel JP and Herrmann FR: Death toll exceeded 70,000 in Europe during the summer of 2003. C R Biol 331: 171-178, 2008.

2. Bouchama A and Knochel JP: Heat stroke. N Engl J Med 346: 1978-1988, 2002

3. Lu KC, Wang JY, Lin SH, Chu P and Lin YF: Role of circulating cytokines and chemokines in exertional heatstroke. Crit Care Med 32: 399-403, 2004.

4. Bouchama A, Ollivier V, Roberts G, Al Mohanna F, de Prost D, Eldali A, Saussereau E, El-Sayed R and Chollet-Martin S: Experimental heatstroke in baboon: Analysis of the systemic inflammatory response. Shock 24: 332-335, 2005.

5. Roberts GT, Ghebeh H, Chishti MA, Al-Mohanna F, El-Sayed R, Al-Mohanna F and Bouchama A: Microvascular injury, thrombosis, inflammation, and apoptosis in the pathogenesis of heatstroke: A study in baboon model. Arterioscler Thromb Vasc Biol 28: 1130-1136, 2008.

6. Bazzoni G and Dejana E: Endothelial cell-to-cell junctions: Molecular organization and role in vascular homeostasis. Physiol Rev 84: 869-901, 2004.

7. Busse R and Fleming I: Vascular endothelium and blood flow. Handb Exp Pharmacol: 43-78, 2006.

8. Toda N, Nakanishi S and Tanabe S: Aldosterone affects blood flow and vascular tone regulated by endothelium-derived NO: Therapeutic implications. Br J Pharmacol 168: 519-533, 2013.

9. Cines DB, Pollak ES, Buck CA, Loscalzo J, Zimmerman GA McEver RP, Pober JS, Wick TM, Konkle BA, Schwartz BS, et al: Endothelial cells in physiology and in the pathophysiology of vascular disorders. Blood 91: 3527-3561, 1998.

10. Michiels C: Endothelial cell functions. J Cell Physiol 196: 430-443, 2003.

11. Minshall RD and Malik AB: Transport across the endothelium: Regulation of endothelial permeability. Handb Exp Pharmacol: 107-144, 2006.

12. Pober JS and Sessa WC: Evolving functions of endothelial cells in inflammation. Nat Rev Immunol 7: 803-815, 2007.

13. Liu Y, Zhou G, Wang Z, Guo X, Xu Q, Huang Q and Su L: NF-кB signaling is essential for resistance to heat stress-induced early stage apoptosis in human umbilical vein endothelial cells. Sci Rep 5: 13547, 2015.

14. Bouchama A, Hammami MM, Haq A, Jackson J and al-Sedairy S: Evidence for endothelial cell activation/injury in heatstroke. Crit Care Med 24: 1173-1178, 1996.

15. Bartel DP: MicroRNAs: Genomics, biogenesis, mechanism, and function. Cell 116: 281-297, 2004.

16. Pepini T, Gorbunova EE, Gavrilovskaya IN, Mackow JE and Mackow ER: Andes virus regulation of cellular microRNAs contributes to hantavirus-induced endothelial cell permeability. J Virol 84: 11929-11936, 2010. 
17. Nicoloso MS, Spizzo R, Shimizu M, Rossi S and Calin GA MicroRNAs-the micro steering wheel of tumour metastases. Nat Rev Cancer 9: 293-302, 2009.

18. Sun HX, Zeng DY, Li RT, Pang RP, Yang H, Hu YL, Zhang Q, Jiang Y,Huang LY, Tang YB, et al: Essential role of microRNA-155 in regulating endothelium-dependent vasorelaxation by targeting endothelial nitric oxide synthase. Hypertension 60: 1407-1414, 2012.

19. Liu J, Zhu G, Xu S, Liu S, Lu Q and Tang Z: Analysis of miRNA expression profiling in human umbilical vein endothelial cells affected by heat stress. Int J Mol Med 40: 1719-1730, 2017.

20. Ashburner M, Ball CA, Blake JA, Botstein D, Butler H Cherry JM, Davis AP, Dolinski K, Dwiqht SS, Eppiq JT, et al: Gene ontology: Tool for the unification of biology. Nat Genet 25 : 25-29, 2000.

21. The Gene Ontology Consortium: The Gene Ontology resource: 20 years and still GOing strong. Nucleic Acids Res 47(D1): D330-D338, 2019.

22. Kanehisa M, Sato Y, Furumichi M, Morishima K and Tanabe, $M$ New approach for understanding genome variations in KEGG. Nucleic Acids Res 47(D1): D590-D595, 2019

23. Kanehisa M, Furumichi M, Tanabe M, Sato Y and Morishima K: KEGG: New perspectives on genomes, pathways, diseases and drugs. Nucleic Acids Res 45(D1): D353-D361, 2017.

24. Kanehisa M and Goto S: KEGG: Kyoto encyclopedia of genes and genomes. Nucleic Acids Res 28: 27-30, 2000.

25. Tang S, Allagadda V, Chibli H, Nadeau JL and Mayer GD: Comparison of cytotoxicity and expression of metal regulatory genes in zebrafish (Danio rerio) liver cells exposed to cadmium sulfate, zinc sulfate and quantum dots. Metallomics 5: 1411-1422, 2013.

26. Tang S, Cai Q, Chibli H, Allaqadda V, Nadeau JL and Mayer GD Cadmium sulfate and CdTe-quantum dots alter DNA repair in zebrafish (Danio rerio) liver cells. Toxicol Appl Pharmacol 272: 443-452, 2013.

27. Livak KJ and Schmittgen TD: Analysis of relative gene expression data using real-time quantitative PCR and the 2(-Delta Delta C(T)) method. Methods 25: 402-408, 2001.

28. Pu Q, Huang Y, Lu Y, Peng Y, Zhang J, Feng G, Wang C, Liu L and Dai Y: Tissue-specific and plasma microRNA profiles could be promising biomarkers of histological classification and TNM stage in non-small cell lung cancer. Thorac Cancer 7: 348-354, 2016.

29. Bibi F, Naseer MI, Alvi SA, Yasir M, Jiman-Fatani AA, Sawan A, Abuzenadah AM, Al-Qahtani MH and Azhar EI: microRNA analysis of gastric cancer patients from Saudi Arabian population. BMC Genomics 17 (Suppl 9): S751, 2016.

30. Ji H, Chen M, Greening DW, He W, Rai A, Zhang W and Simpson RJ: Deep sequencing of RNA from three different extracellular vesicle (EV) subtypes released from the human LIM1863 colon cancer cell line uncovers distinct miRNA-enrichment signatures. PLoS One 9: e110314, 2014

31. Sohn EJ, Won G, Lee J, Lee S and Kim SH: Upregulation of miRNA3195 and miRNA374b mediates the anti-angiogenic properties of melatonin in hypoxic PC-3 prostate cancer cells J Cancer 6: 19-28, 2015.

32. Suresh R, Sethi S, Ali S, Giorgadze T and Sarkar FH: Differential expression of MicroRNAs in papillary thyroid carcinoma and their role in racial disparity. J Cancer Sci Ther 7: 145-154, 2015.

33. Zhou RP, Chen G, Shen ZL and Pan LQ: Cinobufacin suppresses cell proliferation via miR-494 in BGC- 823 gastric cancer cells. Asian Pac J Cancer Prev 15: 1241-1245, 2014.

34. Linnstaedt SD, Walker MG, Parker JS, Yeh E, Sons RL, Zimny E, Lewandowski C, Hendry PL, Damiron K, Pearson C, et al: MicroRNA circulating in the early aftermath of motor vehicle collision predict persistent pain development and suggest a role for microRNA in sex-specific pain differences. Mol Pain 11: 66, 2015.

35. Liu H, Qin H, Chen GX, Liang MY, Rong J, Yao JP and Wu ZK: Comparative expression profiles of microRNA in left and right atrial appendages from patients with rheumatic mitral valve disease exhibiting sinus rhythm or atrial fibrillation. J Transl Med 12: 90, 2014

36. Wang N, Bu R, Duan Z, Zhang X, Chen P, Li Z, Wu J, Cai G and Chen $X$ : Profiling and initial validation of urinary microRNAs as biomarkers in IgA nephropathy. PeerJ 3: e990, 2015.

37. Kantidze OL, Velichko AK and Razin SV: Heat stress-induced transcriptional repression. Biochemistry (Mosc) 80: 990-993, 2015.
38. Kramerov DA and Vassetzky NS: SINEs. Wiley Interdiscip Rev RNA 2: 772-786, 2011.

39. Liu WM, Chu WM, Choudary PV and Schmid CW: Cell stress and translational inhibitors transiently increase the abundance of mammalian SINE transcripts. Nucleic Acids Res 23: 1758-1765, 1995.

40. Caizergues-Ferrer M, Bouche G, Banville D and Amalric F: Effect of heat shock on RNA polymerase activities in Chinese hamster ovary cells. Biochem Biophys Res Commun 97: 538-545, 1980.

41. Mariner PD, Walters RD, Espinoza CA, Drullinger LF, Wagner SD, Kugel JF and Goodrich JA: Human Alu RNA is a modular transacting repressor of mRNA transcription during heat shock. Mol Cell 29: 499-509, 2008.

42. Yakovchuk P, Goodrich JA and Kugel JF: B2 RNA and Alu RNA repress transcription by disrupting contacts between RNA polymerase II and promoter DNA within assembled complexes. Proc Natl Acad Sci USA 106: 5569-5574, 2009.

43. Rougvie AE and Lis JT: The RNA polymerase II molecule at the 5'end of the uninduced hsp70 gene of D. melanogaster is transcriptionally engaged. Cell 54: 795-804, 1988.

44. Gilmour DS and Lis JT: RNA polymerase II interacts with the promoter region of the noninduced hsp70 gene in Drosophila melanogaster cells. Mol Cell Biol 6: 3984-3989, 1986.

45. Yang CC, Ornatsky OI, McDermott JC, Cruz TF and Prody CA: Interaction of myocyte enhancer factor 2 (MEF2) with a mitogen-activated protein kinase, ERK5/BMK1. Nucleic Acids Res 26: 4771-4777, 1998.

46. Kato Y, Zhao M, Morikawa A, Sugiyama T, Chakravortty D, Koide N, Yoshida T, Tapping RI, Yang Y, Yokochi T and Lee JD: Big mitogen-activated kinase regulates multiple members of the MEF2 protein family. J Biol Chem 275: 18534-18540, 2000

47. Kamakura S, Moriguchi T and Nishida E: Activation of the protein kinase ERK5/BMK1 by receptor tyrosine kinases. Identification and characterization of a signaling pathway to the nucleus. J Biol Chem 274: 26563-26571, 1999.

48. English JM, Pearson G, Baer R and Cobb MH: Identification of substrates and regulators of the mitogen-activated protein kinase ERK5 using chimeric protein kinases. J Biol Chem 273: 3854-3860, 1998

49. Watson FL, Heerssen HM, Bhattacharyya A, Klesse L, Lin MZ and Segal RA: Neurotrophins use the Erk5 pathway to mediate a retrograde survival response. Nat Neurosci 4: 981-988, 2001.

50. Nithianandarajah-Jones GN, Wilm B, Goldring CE, Müller J and Cross MJ: ERK5: Structure, regulation and function. Cell Signal 24: 2187-2196, 2012.

51. Oleinik NV, Krupenko NI and Krupenko SA: Cooperation between JNK1 and JNK2 in activation of p53 apoptotic pathway. Oncogene 26: 7222-7230, 2007.

52. Zhong S, Fromm J and Johnson DL: TBP is differentially regulated by c-Jun N-terminal kinase 1 (JNK1) and JNK2 through Elk-1, controlling c-Jun expression and cell proliferation. Mol Cell Biol 27: 54-64, 2007.

53. Chow CW, Dong C, Flavell RA, Davis RJ: c-Jun NH(2)-terminal kinase inhibits targeting of the protein phosphatase calcineurin to NFATc1. Mol Cell Biol 20: 5227-5234, 2000.

54. Li L, Tan H, Yang H, Li F, He X, Gu Z, Zhao M and Su L: Reactive oxygen species mediate heat stress-induced apoptosis via ERK dephosphorylation and Bcl-2 ubiquitination in human umbilical vein endothelial cells. Oncotarget 8: 12902-12916, 2017.

55. Gu ZT, Wang H, Li L, Liu YS, Deng XB, Huo SF, Yuan FF, Liu ZF, Tong HS and Su L: Heat stress induces apoptosis through transcription-independent $\mathrm{p} 53$-mediated mitochondrial pathways in human umbilical vein endothelial cell. Sci Rep 4: 4469, 2014.

56. Zhang S, Liu Y, Wang Z, Liu J, Gu Z, Xu Q and Su L: PAR1-mediated c-Jun activation promotes heat stress-induced early stage apoptosis of human umbilical vein endothelial cells. Mol Med Rep 15: 2595-2603, 2017.

57. Dermott JM, Ha JH, Lee CH and Dhanasekaran N: Differential regulation of Jun N-terminal kinase and p38MAP kinase by Galpha12. Oncogene 23: 226-232, 2004.

58. Raviv Z, Kalie E and Seger R: MEK5 and ERK5 are localized in the nuclei of resting as well as stimulated cells, while MEKK2 translocates from the cytosol to the nucleus upon stimulation. J Cell Sci 117: 1773-1784, 2004.

59. Schmidt C, Peng B, Li Z, Sclabas GM, Fujioka S, Niu J, Schmidt-Supprian M, Evans DB, Abbruzzese JL and Chiao PJ: Mechanisms of proinflammatory cytokine-induced biphasic NF-kappaB activation. Mol Cell 12: 1287-1300, 2003. 
60. Sun W, Vincent S, Settleman J and Johnson GL: MEK kinase 2 binds and activates protein kinase C-related kinase 2 . Bifurcation of kinase regulatory pathways at the level of an MAPK kinase kinase. J Biol Chem 275: 24421-24428, 2000.

61. Sui X, Kong N, Ye L, Han W, Zhou J, Zhang Q, He C and Pan H: p38 and JNK MAPK pathways control the balance of apoptosis and autophagy in response to chemotherapeutic agents. Cancer Lett 344: 174-179, 2014.

62. Xia Z, Dickens M, Raingeaud J, Davis RJ and Greenberg ME: Opposing effects of ERK and JNK-p38 MAP kinases on apoptosis. Science 270: 1326-1331, 1995

63. Kesavan K, Lobel-Rice K, Sun W,Lapadat R, Webb S, Johnson GL and Garrington TP: MEKK2 regulates the coordinate activation of ERK5 and JNK in response to FGF-2 in fibroblasts. J Cell Physiol 199: 140-148, 2004.

64. Nithianandarajah-Jones GN, Wilm B, Goldring CE, Müller J and Cross MJ: The role of ERK5 in endothelial cell function. Biochem Soc Trans 42: 1584-1589, 2014.
65. Wu Y and Chakrabarti S: ERK5 mediated signalling in diabetic retinopathy. Med Hypothesis Discov Innov Ophthalmol 4: 17-26, 2015.

66. Weston CR and Davis RJ: The JNK signal transduction pathway. Curr Opin Genet Dev 19: 142-149, 2007.

67. Xuan P, Han K, Guo Y, Li J, Li X, Zhong Y, Zhang Z and Ding J: Prediction of potential disease-associated microRNAs based on random walk. Bioinformatics 31: 1805-1815, 2015.

68. Chen X, Yin J, Qu J and Huang L: MDHGI: Matrix decomposition and heterogeneous graph inference for miRNA-disease association prediction. PLoS Comput Biol 14: e1006418, 2018.

(i) $(-)$ This work is licensed under a Creative Commons Attribution-NonCommercial-NoDerivatives 4.0 International (CC BY-NC-ND 4.0) License. 